\title{
Predictors of fatal outcome of severe malaria in children of Bhopal, Central India: Retrospective study
}

\author{
Pramila Verma $^{1}$, Shweta Anand ${ }^{2}$, Anju Kapoor ${ }^{3}$ \\ Sri Lanka Journal of Child Health, 2015; 44(1): 17-23
}

\begin{abstract}
Objective: To identify predictors of fatal outcome of severe malaria in children admitted to a tertiary care centre.

Method: This retrospective study was performed at a tertiary health care institution which is a low transmission area of malaria. Confirmed malaria patients (P. falciparum, P. vivax or mixed infection) satisfying one or more of the 2010 WHO criteria for severe malaria were included in the study. Prevalence of fatal outcome due to major symptoms was calculated followed by univariate regression analysis to identify major predictors of fatal outcome.
\end{abstract}

Results: A total of 102 children satisfied WHO criteria of severe malaria and were included in the study. Fatal outcome was seen in 14 children and girls $(71.4 \% ; 10 / 14)$ were more affected as compared to boys $(\mathrm{p}<0.05)$. The risk of fatal outcome was higher $(71.4 \%$; 10/14) among older children (>7years) but this was not a predicting factor for fatal outcome $(p>0.05)$. Fatal outcome was seen more in children with falciparum infestation $(78.5 \%$; 11/14) but this was insignificant $(\mathrm{p}>0.05)$. Univariate regression analysis identified impaired consciousness ( $<<0.05$, RR-0.25, CI-0.10-0.65), shock $(\mathrm{p}<0.001$, RR-0.12, CI-0.052-0.28), spontaneous bleeding $(\mathrm{p}<0.001, \quad$ RR-0.08, CI-0.04-0.16), multisystem involvement $\quad(p<0.05, \quad R R-0.23, \quad$ CI-0.06-0.78), gastrointestinal dysfunction $(\mathrm{p}<0.05, \mathrm{RR}-0.37$, CI0.14-0.99), renal failure $(\mathrm{p}<0.05, \mathrm{RR}-1.54, \mathrm{CI}-0.81-$ $2.95)$ and respiratory distress $(\mathrm{p}<0.001, \mathrm{RR}-0.17$,CI0.07-0.40) as predictors of fatal outcome. Severely anaemic children of severe malaria hardly ever had fatal outcome $(p<0.05)$.

\footnotetext{
${ }_{1}^{1}$ Associate Professor in Paediatrics, Peoples College of Medical Sciences, ${ }^{2}$ Associate Professor in Paediatrics, Chirayu Medical College, ${ }^{3}$ Professor in Paediatrics, Peoples College of Medical Sciences, Bhopal, Madhya Pradesh, India
}

(Received on 15 April 2014: Accepted after revision on 30 May 2014)
Conclusions: Impaired consciousness, shock, spontaneous bleeding, multisystem involvement, gastrointestinal dysfunction, renal failure and respiratory distress were predictors of fatal outcome of severe malaria in children admitted to the tertiary care centre.

(Keywords: Severe malaria; mortality; predictors of fatal outcome)

\section{Background}

Nearly 2.48 million malaria cases are reported annually from South Asia of which $65 \%$ are from India ${ }^{1}$. In India, the National Vector Borne Disease Control Programme (NVBDCP) reported 1.5 million cases and 767 deaths in $2010^{2}$. If untreated, severe falciparum malaria has nearly $100 \%$ mortality $^{3}$. In tropical countries with a high transmission of malaria (hyperendemic areas), severe malaria is predominantly a disease of young children (1 month to 5 years of age $)^{4}$. On the other hand, in places of lower endemicity, viz., South East Asia, children above 5 years and adults suffer from severe falciparum malaria ${ }^{5}$. WHO has described criteria for severe malaria only in plasmodium falciparum ( $\mathrm{P}$. falciparum) patients but now it is proven that plasmodium vivax (P. vivax) and mixed infection ( $\mathrm{P}$. vivax and $P$. falciparum detected in the same child) can also culminate into severe malaria ${ }^{6}$. This retrospective study was undertaken in the Bhopal district of Central India which has reported $7.0 \%$ of all malaria cases and $14.4 \%$ of malaria deaths in 2011 in the country ${ }^{7}$. Substantial research on predictors of mortality of severe malaria has been done in high transmission areas of Africa with few from unstable or low transmission areas of world ${ }^{8,9,10}$.

\section{Objective}

The aim of our study is to find out the predictors of fatal outcome of severe malaria in children admitted to a tertiary care centre of Bhopal in Central India.

\section{Method}

This retrospective study was undertaken in the paediatric department of Peoples College of Medical 
Sciences \& Research Centre, a tertiary care teaching hospital in Bhopal, Central India. Being a teaching hospital and tertiary referral centre, case input is primarily from bordering districts (Vidisha district and Raisen district), Bhopal urban area, Berasia and Gandhi Nagar. The annual parasite index for Bhopal was 1.7 and 0.8 with a slide positivity rate of 1.3 and 0.5 in the years 2003 and 2011 respectively ${ }^{7}$. Case records of confirmed malaria patients admitted between January 2009-December 2012 were retrieved from the medical record department and data was collected in a preformed proforma. Out of 179 children with malaria admitted to the paediatric department during the study period, 102 children satisfied one or more criteria for severe malaria as per World Health Organization (WHO) guidelines $2010^{3}$ and were admitted to the Paediatric Intensive Care Unit. All children aged 12 years or less with confirmed malaria infestation $(\mathrm{P}$. falciparum or $\mathrm{P}$. vivax as mono-infection or $\mathrm{P}$. falciparum and $\mathrm{P}$. vivax in the same patient as mixed infection) either by peripheral smear or by malaria antigen test were included in the study. For inclusion of patients, most criteria of severe malaria ${ }^{3}$ were considered but a few like hyperparasitaemia and hyperlactataemia could not be done in our set up. Also arterial blood gas analysis (ABG) was not affordable to most patients and was done only in a few cases and hence this was not included during analysis in our study. Acute respiratory distress syndrome (ARDS) was seen in fewer children and hence was not included in analysis.

Prior to the commencement of the study the following variables were defined:

Cured: Children who improved clinically and remained stable during hospital stay

Fatal outcome: Children who died and children who left hospital against medical advice (LAMA) after 48 hours of admission and antimalarial treatment in a moribund state with little hope of survival.

Impaired consciousness: Glasgow Coma Score (GCS) less than 5

Shock: Systolic blood pressure (SBP) $<70 \mathrm{~mm} \mathrm{Hg}$ in the presence of cold clammy skin and weak peripheral pulse

Jaundice: Serum bilirubin $>3 \mathrm{mg} / \mathrm{dl}$ along with clinical jaundice.

Clinical haemoglobinuria: Dark-red or blackcoloured urine and negative urine test for red blood cells (RBC) in a G-6-PD negative child

Multiple convulsions: Repeated or prolonged seizures without signs of meningitis

Renal failure: Urine output less than $0.5 \mathrm{ml} / \mathrm{kg}$ per hour and serum creatinine greater than of $1.5 \mathrm{mg} / \mathrm{dl}$.
Malnutrition: Weight $<2$ SDs for age according to Centers for Disease Control and Prevention (CDC) growth charts

Respiratory distress (RD): Tachypnoea (respiratory rate $>40 /$ minute) or retractions (subcostal/ intercostal) or both.

Gastrointestinal dysfunction (GI dysfunction): Patients with concomitant features meeting the definition of severe malaria along with vomiting, refusal to eat or drink and/or abdominal cramps

Spontaneous bleeding: Bleeding from the nose, venipuncture sites, gums, or gastrointestinal tract in the presence of laboratory evidence of disseminated intravascular coagulation (DIC)

Multiple organ dysfunction (MOD): Involvement of 2 or more than 2 organ systems (central nervous system, respiratory, renal, hepatic, gastrointestinal, and haematological disorder)

Haematological disorder: thrombocytopenia, spontaneous bleeding, and DIC but anaemia was excluded from it as defined by Tripathy et al ${ }^{9}$.

Severe anaemia: Haemoglobin $<5 \mathrm{~g} / \mathrm{dl}$

Thrombocytopenia: Platelet count $<150,000$ per $\mathrm{mm}^{3}$,

Raised blood urea: $>40 \mathrm{mg} / \mathrm{dl}$

Raised serum aminotransferase: Serum SGOT $>40$ IU and Serum SGPT >45 IU.

Fatal outcome was considered as the dependent variable and except cure, all the above defined criteria were integrated as independent variables in the univariate regression analysis. All the children with a fatal outcome were having one or more than one independent variable at admission. None of the children were without any potential risk factor.

Analysis was done by SPSS 20.0 software. All clinical indicators that were significant in the univariate logistic regression analysis were included in the multivariate logistic regression analysis, with fatal outcome as the dependent variable. Students' ' $t$ test' was used to compare mean value of continuous variables among cured and fatal outcome children. The Chi-Square test was performed to measure any association between independent and dependent variables and $\mathrm{p}<0.05$ was considered statistically significant.

Permission from institutional research and ethical committee was taken before commencement of the study.

\section{Results}

Data revealed that 179 children were diagnosed and admitted with malaria during the study period, out of 
which $56.9 \%(102 / 179)$ children satisfied the criteria of severe malaria. $86.2 \%(88 / 102)$ children were cured and out of 88 children, 82 children improved and were discharged while 6 clinically stable children left against medical advice (LAMA) due to financial reasons and dissatisfaction. Fatal outcome was observed in $13.7 \%(14 / 100)$ children with severe malaria over four years. Out of 14 children, five died with an overall mortality rate of $4.9 \%(5 / 102)$ whereas 9 children with the moribund condition left the hospital against medical advice after 48 hours of admission during the study period.

Out of 22 independent variables examined, 11 variables were significantly associated with fatal outcome in univariate logistic regression analysis and included in the multivariate logistic regression analysis. These eleven variables were 3 demographic variables, 7 clinical variables and 1 pathological variable. On adjusting confounding factors none of the variables came out to be significant on multivariate logistic regression analysis. On univariate logistic regression analysis, the risk of fatal outcome was higher (71.4\%) among older children who were $>7$ years but statistically age did not emerge to have any significance in predicting death $(\mathrm{p}>0.05)$.

In our study male to female ratio was 1.6:1 but fatal outcome was seen significantly $(\mathrm{p}<0.05)$ more in females. P. falciparum infection was seen in 59 (57.8\%) children, mixed infection in $28(27.4 \%)$ and P. vivax in $15(13.7 \%)$. Five children died and they were all $\mathrm{P}$. falciparum positive; also overall fatal outcome was seen more in children with falciparum infestations $11 / 14(78.5 \%)$ but it was insignificant $(p>0.05)$. Chances of occurrence of complications and fatal outcome were significantly more during initial 3 days of hospitalisation. Three children died within 48 hours while 2 died after 5 days of treatment.

Forty-two percent children of severe malaria had more than one manifestation of severe malaria. The probability of dying from severe malaria increased with an increase in the number of WHO criteria the child presented on admission (Table-I).

Table 1: Demographic variables of children with severe malaria

\begin{tabular}{|c|c|c|c|c|}
\hline Demographic variable & Total No. $n=102$ & Fatal outcome $n=14$ & $\mathbf{X}^{2}$ & p-value \\
\hline $\begin{array}{l}\text { Age } \\
<2 \text { years } \\
2-6 \text { years } \\
7-12 \text { years }\end{array}$ & $\begin{array}{l}20(19.6 \%) \\
35(34.3 \%) \\
47(46.1 \%)\end{array}$ & $\begin{array}{l}02(10.0 \%) \\
02(05.7 \%) \\
10(21.2 \%)\end{array}$ & 4.394 & 0.111 \\
\hline $\begin{array}{l}\text { Gender } \\
\text { Male } \\
\text { Female }\end{array}$ & $\begin{array}{l}63(61.8 \%) \\
39(38.2 \%)\end{array}$ & $\begin{array}{l}04(06.3 \%) \\
10(25.6 \%)\end{array}$ & 7.571 & 0.006 \\
\hline $\begin{array}{l}\text { Species } \\
\text { Falciparum } \\
\text { Vivax } \\
\text { Mixed } \\
\end{array}$ & $\begin{array}{l}59(57.8 \%) \\
15(13.7 \%) \\
28(27.4 \%) \\
\end{array}$ & $\begin{array}{l}11(18.6 \%) \\
02(13.3 \%) \\
01(03.5 \%) \\
\end{array}$ & 3.65 & 0.162 \\
\hline $\begin{array}{l}\text { Duration of stay } \\
<3 \text { Days } \\
>3 \text { Days }\end{array}$ & $\begin{array}{l}11(10.8 \%) \\
91(98.2 \%)\end{array}$ & $\begin{array}{l}08(72.7 \%) \\
06(06.5 \%)\end{array}$ & 36.247 & 0.000 \\
\hline $\begin{array}{r}\text { WHO criteria } \\
1 \\
2 \\
3 \\
4 \\
5\end{array}$ & $\begin{array}{l}59(57.8 \%) \\
24(23.5 \%) \\
13(12.7 \%) \\
05(04.9 \%) \\
01(01.0 \%)\end{array}$ & $\begin{array}{c}04(6.7 \%) \\
03(12.5 \%) \\
04(28.4 \%) \\
02(30.7 \%) \\
01(100 \%)\end{array}$ & 14.824 & 0.005 \\
\hline
\end{tabular}

The probability of fatal outcome among children was $100 \%$ who presented five and more criteria and was $30.7 \%$ those who presented with four criteria compared to those presented with one criterion (6.7\%). Clinical predictors of fatal outcome of severe malaria in children are shown in Table 2 . 
Table 2: Clinical predictors of fatal outcome of severe malaria in children (Univariate regression analysis: Adjusted odds ratio)

\begin{tabular}{|l|c|c|c|c|c|c|}
\hline \multicolumn{1}{|c|}{ Clinical Variable } & $\begin{array}{c}\text { Prevalence } \\
(n=102)\end{array}$ & $\begin{array}{c}\text { Fatal outcome } \\
(n=14)\end{array}$ & $\begin{array}{c}\text { Expired/ } \\
\text { LAMA }\end{array}$ & $\begin{array}{c}\text { Relative Risk } \\
(\mathbf{9 5 \%} \text { CI) }\end{array}$ & $\boldsymbol{X}^{2}$ & $\boldsymbol{P}$ value \\
\hline Impaired consciousness & $21(20.6 \%)$ & $7(33.3 \%)$ & $3 / 4$ & $0.25(0.10-0.65)$ & 8.58 & 0.003 \\
\hline Multi organ dysfunction & $47(46.1 \%)$ & $11(23.4 \%)$ & $5 / 6$ & $0.23(0.06-0.78)$ & 6.89 & 0.009 \\
\hline Multiple convulsions & $20(19.6 \%)$ & $5(20.0 \%)$ & $2 / 3$ & $0.43(0.16-1.16)$ & 2.67 & 0.102 \\
\hline Renal failure & $7(6.9 \%)$ & $3(42.8 \%)$ & $1 / 3$ & $1.54(0.81-2.9)$ & 5.38 & 0.020 \\
\hline Spontaneous bleeding & $6(5.9 \%)$ & $6(100.0 \%)$ & $3 / 3$ & $0.08(0.04-0.16)$ & 40.07 & 0.000 \\
\hline Gastrointestinal dysfunction & $34(33.3 \%)$ & $8(23.5 \%)$ & $3 / 5$ & $0.37(0.14-0.99)$ & 6.89 & 0.048 \\
\hline Shock & $11(10.8 \%)$ & $7(63.6 \%)$ & $3 / 4$ & $0.12(0.05-0.28)$ & 25.93 & 0.000 \\
\hline Clinical haemoglobinuria & $20(19.6 \%)$ & $3(15.0 \%)$ & $2 / 1$ & $0.89(0.27-2.90)$ & 0.03 & 0.853 \\
\hline Respiratory distress & $9(8.8 \%)$ & $5(55.5 \%)$ & $4 / 1$ & $0.17(0.07-0.40)$ & 14.58 & 0.000 \\
\hline Malnutrition & $47(46.1 \%)$ & $8(17.0 \%)$ & $4 / 4$ & $0.64(0.24-1.71)$ & 0.80 & 0.371 \\
\hline
\end{tabular}

While clinical haemoglobinuria, malnutrition and multiple convulsions resulted in fatal outcome in $15 \%, 17 \%$ and $20 \%$ of children, these variables did not predict fatal outcome (Table 2).

Probability of dying or fatal outcome increased if the child had impaired consciousness (GCS score $<5$ ). One child who presented with cerebral malaria as an individual manifestation had a fatal outcome without any other major manifestations. Probability of fatal outcome increased if two or more systems were involved $(\mathrm{p}<0.05)$ in the same child with severe malaria. Shock resulted in fatal outcome in $63.6 \%$ of children of whom one child died within 24 hours, 2 died after 5 days and 4 LAMA. Respiratory distress
(RD) was observed in $8.8 \%$ children of whom four had superimposed pneumonia along with severe malaria that resolved with treatment and $55.5 \%$ had fatal outcome. Out of the 4 children who died 3 developed ARDS after packed RBC transfusion.

Severe anaemia was observed in $68.6 \%$ of children, mean haemoglobin was $5.2 \mathrm{~g} / \mathrm{dl}$ (lowest haemoglobin was $1.5 \mathrm{~g} / \mathrm{dl}$ ), and more associated with children those who were cured (with antimalarial drugs and blood transfusion) and got discharged $(\mathrm{p}<0.05)$ (Tables $3 \&$ 4). In our study population severe anaemia was infrequently seen in children those who proceeded to fatal outcome.

Table 3: Mean values of continuous variables of severe malaria children

\begin{tabular}{|l|c|c|c|c|c|}
\hline $\begin{array}{c}\text { Laboratory } \\
\text { variable }\end{array}$ & $\begin{array}{c}\text { Range } \\
\mathbf{( n = 1 0 2})\end{array}$ & $\begin{array}{c}\text { Mean value } \\
(\mathbf{n = 1 0 2})\end{array}$ & $\begin{array}{c}\text { Mean value of } \\
\text { cured children }\end{array}$ & $\begin{array}{c}\text { Mean value of fatal } \\
\text { outcome }(\mathbf{n}=\mathbf{1 4})\end{array}$ & $\begin{array}{c}p \text { - } \\
\text { value }\end{array}$ \\
\hline Haemoglobin & $1.5-11.6 \mathrm{~g} / \mathrm{dl}$ & $5.3 \mathrm{~g} / \mathrm{dl}$ & $5.2 \mathrm{~g} / \mathrm{dl}$ & $5.88 \mathrm{~g} / \mathrm{dl}$ & 0.362 \\
\hline $\begin{array}{l}\text { Total leucocyte } \\
\text { count }\end{array}$ & $900-65,000 / \mathrm{mm}^{3}$ & $10,895 / \mathrm{mm}^{3}$ & $8184.4 / \mathrm{mm}^{3}$ & 12870.6 & 0.023 \\
\hline Serum bilirubin & $0.33-36 \mathrm{mg} / \mathrm{dl}$ & $2.92 \mathrm{mg} / \mathrm{dl}$ & $2.86 \mathrm{mg} / \mathrm{dl}$ & $3.33 \mathrm{mg} / \mathrm{dl}$ & 0.7892 \\
\hline Platelet counts & $9300-463,000 / \mathrm{mm}^{3}$ & $101,080 / \mathrm{mm}^{3}$ & $107,820 / \mathrm{mm}^{3}$ & $56,150 / \mathrm{mm}^{3}$ & 0.084 \\
\hline Blood urea & $10.6-233 \mathrm{mg} / \mathrm{dl}$ & $61.1 \mathrm{mg} / \mathrm{dl}$ & $55.7 \mathrm{mg} / \mathrm{dl}$ & $102.0 \mathrm{mg} / \mathrm{dl}$ & 0.0296 \\
\hline Serum SGOT & $17-675 \mathrm{IU}$ & $112.4 \mathrm{IU}$ & $106.80 \mathrm{IU}$ & $158.0 \mathrm{IU}$ & 0.283 \\
\hline Serum SGPT & $16-1431 \mathrm{IU}$ & $114.3 \mathrm{IU}$ & $108.23 \mathrm{IU}$ & $153.03 \mathrm{IU}$ & 0.650 \\
\hline
\end{tabular}


Table 4: Laboratory predictors of fatal outcome of severe malaria in children (Univariate regression analysis: Adjusted odds ratio)

\begin{tabular}{|l|c|c|c|c|c|c|}
\hline Laboratory variable & $\begin{array}{c}\text { Prevalence } \\
(n=102)\end{array}$ & $\begin{array}{c}\text { Fatal outcome } \\
(n=14)\end{array}$ & $\begin{array}{c}\text { Expired } \\
\text { /LAMA }\end{array}$ & $\begin{array}{c}\text { Relative Risk } \\
\mathbf{9 5 \%} \text { CI }\end{array}$ & $\boldsymbol{X}^{\mathbf{2}}$ & $\boldsymbol{P}$ value \\
\hline Severe anaemia & $70(68.6 \%)$ & $6(8.5 \%)$ & $1 / 5$ & $2.917(1.103-7.712)$ & 5.005 & 0.025 \\
\hline Jaundice & $15(14.7 \%)$ & $3(20.0 \%)$ & $3 / 0$ & $1.092(0.837-1.424)$ & 0.585 & 0.444 \\
\hline Thrombocytopenia & $77(75.5 \%)$ & $11(14.2 \%)$ & $4 / 7$ & $0.840(0.254-2.773)$ & 0.083 & 0.773 \\
\hline Raised blood urea & $33(32.4 \%)$ & $5(15.1 \%)$ & $1 / 4$ & $0.861(0.313-2.367)$ & 0.084 & 0.772 \\
\hline Raised Serum SGOT & $54(52.9 \%)$ & $6(11.1 \%)$ & $1 / 5$ & $1.500(0.560-4.015)$ & 0.662 & 0.416 \\
\hline Raised serum SGPT & $40(39.2 \%)$ & $5(12.5 \%)$ & $0 / 5$ & $1.161(0.419-3.215)$ & 0.083 & 0.773 \\
\hline
\end{tabular}

Maximum drop in platelet counts was seen in children of fatal outcome group although thrombocytopenia did not emerge as a predictor of fatal outcome $(\mathrm{P}>0.05)$. Leucocytosis was seen significantly in fatal outcome children $(p<0.05)$. Jaundice was seen in 15 children of whom three died but they also had one or more clinically significant manifestations. In all children jaundice was associated with hepatitis (Highest rise in SGPT and SGOT was $1431 \mathrm{IU}, 675$ IU respectively). Raised blood urea $(>40 \mathrm{mg} / \mathrm{dl})$ was seen in 33 children with severe malaria (maximum rise in urea was $233 \mathrm{mg} / \mathrm{dl}$ ) while more than $100 \mathrm{mg} / \mathrm{dl}$ was seen in 5 children.

\section{Major predictors of fatal outcome}

Various combinations of clinical predictors of severe malaria in children were included in Chi Square analysis to find out significant combinations that resulted in fatal outcome. These combined or overlapping presentations that were seen in our patients were MODs, DIC, shock, impaired consciousness, renal failure, respiratory distress and gastrointestinal dysfunction.

\section{Overlapping and associations of significant} predictors

MODs, respiratory distress gastro-intestinal dysfunction, renal failure and shock as a single major manifestation was seen in 17, 1, 17, 1 and 3 children respectively. They all improved and were discharged from hospital. However, DIC and shock as a primary and single manifestation were not seen in any children but always presented as overlapping syndrome. MODs in combination with any one or more features (impaired consciousness, DIC, shock, renal failure, respiratory distress) resulted in fatal outcome in $78.5 \%(11 / 14)$ children $(p<0.05)$. Shock in combination with MODs resulted in fatal outcome in $50 \%(7 / 14)$ children. Impaired consciousness as a single manifestation resulted in mortality in one child while it caused fatal outcome when overlapped with MODs in $42.8 \%(6 / 14)$ children. Spontaneous bleeding (DIC) in combination with other features resulted in mortality in $42.8 \%(6 / 14)$ children, while
DIC in combination with shock caused mortality in $66.6 \%(4 / 6)$. Respiratory distress in combination with other MODs was responsible for fatal outcome in $35.7 \%(5 / 14)$ children. GI dysfunction was seen in combination with other major manifestations in $57.1 \%(8 / 14)$ children and they all culminated in fatal outcome. MODs and GI dysfunction together were seen in $42.8 \%(6 / 14)$ children and resulted in improved outcome.

\section{Discussion}

In the current study clinical predictors of fatal outcome of severe malaria were impaired consciousness, shock, spontaneous bleeding, MOD, GI dysfunction, renal failure and respiratory distress. A few predictors like impaired consciousness, shock, respiratory distress and MOD were associated with poor outcome in many studies ${ }^{8-12}$. Our results show that age is not predictive of fatal outcome in low transmission area although fatal outcome occurred more in children who were older than seven years. This is in contrast to the study by Imbert et al ${ }^{10}$ and Tripathy et $\mathrm{al}^{9}$ who found that mortality is more in children who were younger than 2 years.

Boys got admitted one and a half times more than girls during study period but fatalities were seen 2.5 times more often in girls. It may be due to increased probability of boys having an outdoor exposure, although a genetic basis cannot be ruled out. This is opposite to results shown by Singh et al from Northern India ${ }^{13}$. In Madhya Pradesh both P. vivax and $P$. falciparum species are prevalent and the results of our study show that risk of P. falciparum malaria was four times higher compared with that of P. vivax malaria. This is in accordance with a previous report by Singh et al in Panna district of Madhya Pradesh from 2003-2005 ${ }^{14}$. Results of our study also reveal that a fatal outcome was seen more in children of P. falciparum while children with P. vivax and mixed infection had less severe manifestations. These findings are similar to the study conducted in Mohapatra, India ${ }^{15}$. 
It is evident from our study that the mortality rate is directly proportional to the number of WHO criteria present at the time of admission and number of organs involved (MODs). This is comparable to other studies ${ }^{16-18}$. Incomplete or partially treated children who received less than three days of antimalarial treatment were also at increased risk for fatal outcome $(p<0.05)$. This is understandable as the majority of deaths $(3 / 5)$ among patients with malaria occur within the first 48 hours after their hospital admission, and rapid provision of supportive care at this point could be lifesaving. In our study impaired consciousness was seen in $21(20.6 \%)$ children and one-third of them had fatal outcome. This is in contrast to results of various studies in India where coma was found responsible for the majority of deaths in children with severe malaria ${ }^{9,} 11,19$. In our study children with renal failure were more than 7 years of age, which is similar to results shown by Satpaty et al from India ${ }^{11}$. Acute renal failure from malaria-induced acute tubular necrosis is rare in children from any cause and is very rarely reported in children with severe malaria ${ }^{20}$.

Severe anaemia was a predominant manifestation in our study group which is in agreement with the observations by Jain et al from Jabalpur ${ }^{21}$. As shown by Imbert et al from Senegal, high prevalence of severe anaemia in young children depends more on age and parasitaemia than on transmission level ${ }^{10}$. However, it is strongly predictive of non-fatal outcome in severe malarial children in our study group. This is similar to that seen in children of Allahabad ${ }^{19}$ but inconsistent with results shown by Seidlein et al who found no association of severe anaemia with outcome ${ }^{8}$. In our study population RD was seen only in few children and most of them had pneumonia. Pulmonary oedema was not seen in any child at admission but 3 severely anaemic children developed ARDS after packed RBC transfusion. Association of transfusion with the development of ARDS in children has not been described but in adults there are many studies which support $\mathrm{it}^{22}$.

All patients who had fatal outcome in our study had more than one feature (MOD, impaired consciousness, shock, DIC and respiratory distress) as overlapping presentations except for one child who died as an individual manifestation with impaired consciousness. Fatal outcome is unusual among patients presenting with individual manifestations of MOD, GI dysfunction, anaemia, thrombocytopenia, jaundice, haemoglobinuria or hepatitis as the one and only essential severity decisive factor. Although these manifestations are not decisive of severe malaria (WHO criteria) they are most of the time associated with fatal outcome. It is important that if the patient is having MOD, GI dysfunction, severe anaemia, thrombocytopenia, jaundice, haemoglobinuria or hepatitis as initial manifestations, child should be managed aggressively so as to prevent shock, DIC, respiratory distress or cerebral malaria.

A few variables like DIC, MOD and ARDS in children with severe malaria are commonly associated with poor outcome but there are scanty studies that have considered these variables so there is a need for more exploration on these predictors.

\section{Conclusions and recommendations}

Impaired consciousness, shock, spontaneous bleeding, multisystem involvement, gastrointestinal dysfunction, renal failure and respiratory distress were predictors of fatal outcome of severe malaria in children admitted to the tertiary care centre.

Since this was a retrospective study with small sample size and critically sick LAMA patients (their final outcome not known) were also included in the study. In our study none of the variable was significant on multivariate regression analysis. With this data and analysis, results cannot be relied on completely. There is a need for further prospective studies in this region so as to ascertain predictors of fatal outcome of severe malaria.

\section{References}

1. World Health Organization. World malaria report. Geneva: WHO; 2012.

2. Statewise malaria situation during 2009-2012. National Vector Borne Disease Control Program. 22-Sham Nath Marg Delhi-110054. Available from: http://nvbdcp.gov.in/malaria

3. World Health Organization. The guidelines for the treatment of malaria. 2nd Ed. Geneva: WHO; 2010 .

4. Trampuz A, Jereb M, Prabhu RM. Clinical review: Severe malaria Critical Care 2003; 7(4): 315-23. http://dx.doi.org/10.1186/cc2183

5. World Health Organization Severe falciparum malaria. Transactions of the Royal Society of Tropical Medicine and Hygiene 2000; 94 (Suppl 1): S1-S90. http://dx.doi.org/10.1016/S0035-9203(00)90300$\underline{6}$ 
6. Mail BG, D'Acremont V, Rare L, Bee K, Reeder JC, Alpers MP, et al. Plasmodium vivax and mixed infections are associated with severe malaria in children: A prospective cohort study from Papua New Guinea Published: June 17, 2008 DOI: 10.1371/journal. Pubmed. 0050127

7. Annual reports on malaria 2003-2011. District malaria centre Bhopal (M.P.)

8. von Seidlein L, Olaosebikan R, Dondorp AM. Predicting the clinical outcome of severe falciparum malaria in African children: Findings from a large randomized trial Clinical Infectious Diseases 2012; 54(8): 1080-90. http://dx.doi.org/10.1093/cid/cis034

9. Tripathy R, Parida S, Das L, Mishra DP, Tripathy D, Das MC, et al. Clinical manifestations and predictors of severe malaria in Indian Children. Pediatrics 2007; 120; e454e460. http://dx.doi.org/10.1542/peds.2006-3171

10. Imbert P, Sartelet I, Rogier C, Ka S, Baujat G, Candito D. Severe malaria among children in a low seasonal transmission area, Dakar, Senegal: influence of age on clinical presentation. Transactions of the Royal Society of Tropical Medicine and Hygiene 1997; 91:22- 4. http://dx.doi.org/10.1542/peds.2006-3171

11. Satpathy SK, Mohanty N, Nanda P, Samal G. Severe falciparum malaria. Indian Journal of Pediatrics 2004; 71:133- 5. http://dx.doi.org/10.1007/BF02723094

12. Mockenhaupt FP, Ehrhardt S, Burkhardt J, et al. Manifestation and outcome of severe malaria in children in Northern Ghana. American Journal of Tropical Medicine and Hygiene 2004; 71:167- 72 .

13. Singh R, Kumar S, Rana SK, Thakur B, Singh SP. Journal of Clinical and Diagnostic Research 2013; 7(10): 2234-7.

14. Singh N, Chand SK, Mishra AK, Bharti PK, Singh MP, Ahluwalia TP, et al. Epidemiology of malaria in an area of low transmission in central India. American Journal of Tropical Medicine and Hygiene 2006; 75(5):812-6.
15. Mohapatra MK, Dash LK, Barih PK, Karua PC. Profile of mixed species (Plasmodium vivax and falciparum) malaria in adults Journal of the Association of Physicians of India 2012; 60:204.

16. Huda SN, Shahab T, Ali SM, Afzal K, Khan HM. A comparative clinical trial of artemether and quinine in children with severe malaria Indian Pediatrics 2003; 40:939- 45.

17. Kalinga A, Mayige M, Kagaruki G, Shao A, Mwakyusa B, Jacob F. Clinical manifestations and outcomes of severe malaria among children. Tanzania Journal of Health Research 2012; 14(1): http://dx.doi.org/10.4314/thrb.v14i1.2

18. Khilnani P, Sarma D, Zimmerman J. Epidemiology and peculiarities of pediatric multiple organ dysfunction syndrome in New Delhi, India. Intensive Care Medicine 2006; 32(11):1856-62. http://dx.doi.org/10.1007/s00134-006-0373-5

19. Kumar A, K.shrivastava A, Taksande A, Singh $D$, Rai R. Severe P. falciparum malaria in children in a tertiary care center of Allahabad region of India. The Internet Journal of Pediatrics and Neonatology Volume 12 No. 1.

20. Olowu WA, Adelusola KA. Pediatric acute renal failure in southwestern Nigeria. Kidney International 2004; 66:1541-8. http://dx.doi.org/10.1111/j.15231755.2004.0091 8.x

21. Jain V, Nagpal AC, Joel PK, Shukla M, Singh MP, Gupta RB, et al. Burden of cerebral malaria in Central India (2004-2007). American Journal of Tropical Medicine and Hygiene 2008; 79: 636 $-42$.

22. Gong MN, Thompson BT, Williams P, Pothier L, Boyce PD, Christiani DC. Clinical predictors of and mortality in acute respiratory distress syndrome: potential role of red cell transfusion. Critical Care Medicine 2005; 33: $1191-8$. http://dx.doi.org/10.1097/01.CCM.0000165566.8 $\underline{2925.14}$ 